\title{
Studies on the character and staining of fibrin
}

\author{
A. C. LENDRUM, D. S. FRASER, W. SLIDDERS, AND R. HENDERSON
}

From the University of St. Andrews Department of Pathology, Royal Infirmary, Dundee

There are few satisfactory staining methods for fibrin. There are even fewer clear definitions of the material for which these methods are commended.

In sections of tissue the older pathologists recognized three phenomena to which they gave the name fibrin. These were: $(a)$ The networks and more solid masses within blood vessels that formed a large part of the substance of what had been seen with the naked eye as a thrombus; $(b)$ the extravascular networks of somewhat comparable structure associated with acute inflammation and seen in the interstitial tissue, in the arachnoid space, or in pneumonic alveoli; and $(c)$ the more solid and plaque-like masses occurring on serous surfaces. When observations came to be made on the hyaline eosinophilic fibrin-like material found to be present in pathological processes other than simple coagulation and the pyogenic lesions, the names given to it were curiously varied. It is a measure of this vagueness that in tissues from rheumatic disease of the heart the eosinophilic deposit on the epicardial surface was called 'fibrin', a comparable deposit in the superficial layers of the mitral valve was called 'platelets', and the eosinophilic and slightly refractile network of the reticular Aschoff body was called 'fibrinoid'. Within recent years, almost contemporaneously with the falling incidence of acute pyogenic inflammation, there has been an increasing recognition and experience of this fibrin-like material which, with the classical methods for staining fibrin, gives varied degrees of positiveness; typical examples are the intra-alveolar networks in the lungs of subjects with uraemia or cardiac failure, and the deposits of hyaline materials within the walls of blood vessels ('fibrinous vasculosis', Lendrum, 1955, 1956) as seen in systemic and pulmonary hypertension, in areas of infarction, and in the diseases which for want of more exact knowledge we group under the term 'sensitization'. It is fashionable still to call this material 'fibrinoid' but the new methods for demonstrating fibrin described below all give a sharp, contrasty staining reaction with this material, a finding which encourages us to abjure this melodious but misleading term.

The fact of identical staining reaction on these Received for publicaticn 6 November 1961. various substances, even with a range of quite diverse methods, is still no proof of chemical identity, nor can one obtain any point of reference outside the field of paraffin-embedded sections of tissue that justifies our concluding that the methods are specific for fibrin as such. Thus, for example, the apparent specificity of staining reaction obtainable on sections of a fibrinous exudate on the pleura is not obtained if we take a network of fibrin from coagulation of blood in vitro, fix it as if a block of tissue, and cut and stain paraffin sections. Within the restricted field of the histopathologist the only available rock to which we can anchor our ideas is the thrombus in the luman of vessels in the sections. Other substances that give a similar and well-defined staining reaction demand critical scrutiny in the light of what we know or can reasonably guess about the genesis of the substance under study. The sensible observer surely does not confine his observation to a colour reaction. Among the other criteria available to aid assessment of the substance is its structure but this taken by itself has not proved adequate; thus, there is little doubt that the term fibrinoid was adopted by some observers because the deposition of fibrin within the close texture of an arterial wall produced there the colour reaction of fibrin but lacked the fibrillar character of fibrin. More extensive study seems to us to show that when the fibrinogen-carrying plasma travelled further into the wall and came to reach the looser tissues of the perivascular area it deposited there a fibrin-staining substance in a fibrillar and net-like arrangement quite characteristic of what we may call standard fibrin. Our concept of the process thus conditions our interpretation of the appearances, as foreknowledge of the story allows us to recognize the characters on the stage. Difficulties still face the informed observer and one he meets early in pathology is when he sees in a section a substance he feels ought, on the basis of its structure, to have given a positive colour reaction for fibrin. This is not new; indeed when studying pneumonic lungs with Weigert's stain for fibrin, many a pathologist in the past must have experienced this, wondered about technical failure in the staining, and soothed himself by the comforting view that the tissue was not fresh. 
Others may well have thought that the age of the fibrin affected its affinity for the various dyes, especially as the two classical methods, Weigert's and phosphotungstic-haematoxylin, by revealing a certain parallelism in the affinity or disinterestedness of what was presumed to be fibrin, seemed thereby to eliminate the possibility of technical failure.

Our own studies have had a dual character in that evolution of the new staining methods was under way at the same time as a personal study (A.C.L.) on fibrinous vasculosis. In the latter condition it was possible to observe deposits of fibrin unaffected by the complicating lysis that accompanies infective processes, and it seemed reasonable to presume that in the absence of fibrinolytic effect the fibrin survived longer, and thus middle-aged and elderly fibrins were available for study. The time sequence can of course merely be surmised, but if sufficient sections are studied it is possible to see in one group of sections a material staining as fibrin which, on the basis of its morphological characters and its site, especially if the latter should be unusual and consistent from case to case, can be equated with a material, in other sections, which stains as cell-free collagen. The validity of the hypothesis (Lendrum, $1961 \mathrm{~b})$ is strengthened if a similarly situated and structurally identical material can be found showing also an intermediate or mixed affinity for any two dyes that designate on the part of one, fibrin and of the other, pseudo-collagen. This transition is readily available for study in the kidney of diabetics with hypertension, where the process is common and widespread, and seems to be compressed in time and so is frequently present in the whole range of transition even within the narrow bounds of a single section. To allow comparison from case to case, it is of the utmost importance to plan the fixation and processing of the tissues with this study in mind; fortunately our particular fixative and processing techniques also provide excellent general histological preparations, and so, with the warning that the material is unsuitable for the enzymatic forms of histochemistry, we give full details in a subsequent section. The possibility of assessing the age of fibrin will be discussed later in this section.

The study of fibrin occurring in fibrinous vasculosis must be accompanied by three cautions. First, elastic tissue commonly resembles middle-aged fibrin in its affinity for dye, but this can be resolved by a primary staining of the elastica with Weigert's original or modified methods or by synthetic orcein. Secondly, in lipaemic cases (e.g., some diabetics) the peripheral plasma in small arteries has an affinity for dye rather like that of fibrin; this similarity is also seen in the pulmonary exudate of some cases of endogenous lipid pneumonia (Morgan 1952). Thirdly, the fibrinogen escaping from the lumen and precipitated intramurally as fibrin form a spongework primarily on the inner face of th\& internal elastic lamina, and in this situation acts as a filtering layer to trap and concentrate other sub stances leaking out from the plasma. This is well see in the mixture of fibrin and fat occurring in some hypertensive arterioles, especially from diabetie patients; in similar manner, in those conditions where abnormal proteins are present in the plasmat for example, in systemic lupus erythematosus oct cryoglobulinaemia, the fibrin filter may well be. clogged by these abnormal proteins. Because of this 'escape' into the wall by substances other tha $\overrightarrow{7}$ fibrinogen, it is suggested that the term 'fibrinouger vasculosis' be replaced by the term suggested by $D$ है Harry Goldblatt, 'plasmatic vasculosis'. Thesê deposited admixtures, of fibrin and fat certainlye and of fibrin and other proteins presumably, do in fact bring about slight differences in the stainin reaction of the adulterated fibrin, but in the cases of lupus erythematosus and of cryoglobulinaemia the colour differences are not enough to permit more than the first step to diagnosis-suspicion. Recent studies on amyloid disease have made it seer fairly certain that it is also a plasmatic vasculgis, and the precipitated accumulation in the intimsic amyloid substance is sometimes, in the kidney least, accompanied by deposition of fibrin; this is io a layered formation and the fibrin has been seen only in small amount, but it is quite distinct becaus the 'coarse texture' of the amyloid material has $a \overrightarrow{8}$ affinity for dyes of very different molecular type from those that stain fibrin. The demonstration of othex proteinous substances from the plasma is perhaps impossible with ordinary staining methods, but the may well be entangled and accumulated in a fibrin or amyloid layer, and thereby susceptible to demong stration by the fluorescent techniques.

The meaning of the term 'fibrin' cannot be stated with any physical or chemical certainty within the context of microscopical observation of tissue sections stained by what may be calle classical methods. Fluorescent microscopy and the. electron microscope have thus far not revealed whiff they surely promise, although the former method has already given suggestive evidence that bot hyaline in vessel walls and 'fibrinoid' are in some way related to fibrin (Crawford and Woolf, 1960 Nonetheless, we feel that the consistency of of methods is enough to justify our using the ter⿳⺈⿴囗十 fibrin for the materials that give the specific reaction and are of a shape and situation compatible with oup working hypotheses about the diseases concerne This reservation is particularly necessary because off 
our findings in the staining of intracellular granules. We believe that the so-called 'acidophil' granules of the pituitary (Slidders, 1961b) have a structural density or polymolecular texture very close to that of newly precipitated fibrin, as do also some of the inclusion bodies of giant-cell pneumonia and this is manifest by their affinity for dyes with a fairly small molecule (see Lendrum, 1961a); moving along the scale we find the affinity for dyes of somewhat larger molecular size shown by pituitary basophils resembles roughly that shown by middle-aged fibrin, by some of the Russell bodies of cancer cells, by the globular bodies of plasma cells, by some of the granules of renal tubule cells in cases with albuminuria, by the inclusions in alveolar phagocytes in lungs the seat of intra-alveolar fibrinous deposits, and by the inclusions in the lungs of ferrets inoculated with canine distemper virus. ${ }^{1}$

Staining reactions for fibrin are, according to our ideas, dependent on the 'fine structure' of the material rather than on a simple chemical reaction as we ordinarily know it; this fine structure, as we have implied above, probably undergoes changes with age and thus what may be called the entanglement of a suitable dye depends on our finding dyes of the right molecular size to match the succeeding structural changes in the ageing fibrin. The newest fibrin likely to be found in sections presumably is the fine network of post-mortem fibrin within the lumen of vessels; much of this with the MSB (Martius, Scarlet, and Blue) method (see subsequent section) takes a yellow stain, as do the erythrocytes, a finding that is comparable with the yellow staining of pituitary alpha cells and erythrocytes with the orange-fuchsin-green method of Slidders (see below). A slightly older fibrin has been seen as tiny lozenges in the wall of glomerular arterioles in the case of a child who died 16 hours after resection of an aortic coarctation (Lendrum, 1961b); we concluded that this plasmatic vasculosis had been produced by the subjection of the renal vessels to a novel high pressure at the time of the operation and for some unknown time thereafter until shock supervened. These lozenges take a bright red stain with the MSB method, comparable with the red staining of pituitary basophils with the analogous orangefuchsin-green method. With the 44/41 Masson method the intravascular network is red and the lozenges blue; with picro-Mallory $\mathrm{V}$ both are red. If we now turn to examples of plasmatic vasculosis in hypertensive diabetic kidneys, we see in arterial and arteriolar walls and in the intercapillary zones of the glomeruli, deposits that with MSB are strongly red, deposits that show a fading red with pale blue

${ }^{1}$ Kindly supplied to us by the Veterinary School, University of Glasgow. appearing, deposits that are hyaline, acellular and blue, and finally in the intercapillary deposits (the so-called Kimmelstiel-Wilson glomerulus) the advent of nuclei within the blue mass. From a study of several such cases we believe that these different appearances as listed are in fact a time sequence, and it is probably a confirmation both of this chronometry and of our views on structural change that we find the MSB method giving the red staining of fibrin in material that with picro-Mallory $\mathrm{V}$ is now blue, while with what we regard as still older fibrin the 44/41 Masson still stains it as fibrin although all the other methods stain it as collagen. The gradual change in fibrils from a positive reaction for fibrin to a reaction for collagen, without any other visible alteration in the fibrils, has been noted by many workers using the older methods; two at least of our methods are capable of revealing the fibrinous origin of fibrils and material too old to react with the classical methods, but the studies of Crawford and Woolf (1960) with fluorescent antibodies suggest that their method can at even later stages of the metamorphosis still indicate a fibrinous origin for this apparent collagen. We may well ask, At what age must fibrin resign the name? Having, as we hope, expunged the term fibrinoid, we are now in the unhappy position of seeking a name for the material which stains as collagen, at different ages depending on the method used, and yet clearly owes its origin to an age change occurring in fibrin. 'Pseudo-collagen' is possibly reasonable, even though this carries the risk of hearing colleagues discoursing on pseudo-collagen diseases. The further changes in the pseudo-collagen are somewhat beyond the purpose of this review, but although it is clear, for example in the intercapillary masses in the diabetic glomerulus, that the acellular mass of metamorphosed fibrin becomes cellular after the stage of pseudo-collagen, it sometimes happens that calcium is deposited in the mass. This occurs particularly in the small vessels of the lung and possibly more commonly in children, and in cases from other geographical regions, for example, from regions with hard water, as Dr. D. A. Leslie of Sunderland suggested to one of us (A.C.L.); our impression is that the calcification occurs at a stage when the fibrin can still be stained with picro-Mallory and thus could be regarded as middle-aged.

The new methods are modified applications of two established principles. Arising out of Mallory's original and quite unexplained use of phosphotungstic acid in his trichromic method, there has been a proliferation of empirical variations and but small study of the way the phosphotungstic acid contributes to the result. Such work as there was is discussed by Baker (1958) who contributes the fertile 
remark, 'If an Irish bull be permissible, one may say shortly that phosphomolybdic acid acts as a colourless acid dye (for it scarcely colours the tissues)'. This idea was followed by one of us (W.S.) and use was also made of the fact (Venkataraman, 1952) that a water-soluble dye dissolved in alcohol then behaves as if it were of a smaller molecular size. It was found that phosphotungstic acid, which we regard as better than phosphomolybdic with human tissue, if dissolved in alcohol now behaved as if it also were of a smaller molecular size and thereby could in a given time enter into more compact structures than the aqueous solution could, for example, into erythrocytes. This brief statement does scant justice to a principle that in its first application produced the orange-fuchsin-green method, a remarkably specific and almost automatic stain for the anterior pituitary (Slidders, 1961b). To make a stain for fibrin on this principle, we found it profitable to change the first, the molecularly smallest dye, the second or middle-sized dye, and the last and largest (collagen-staining) dye; combining theory and empiricism we reached the method described below as MSB and this we have found to have something of the automatic virtue of the orange-fuchsin-green method. Indeed this has virtually replaced the picroMallory V method (see below) which in the hand of the knowledgeable artist is still of value, but only the really skilled will better the results obtained by a reasonable artisan using the MSB method. The latter method has another advantage in that it stains distinctively somewhat older fibrin than is shown by the picro-Mallory $\mathrm{V}$ method.

Another application of Slidders' principle arose from its conjunction with a variant of the Masson method, under study at the same time. This variant, the 44/41 Masson, was itself the result of combining as the first dye a brighter, purer red than acid magenta, namely Brilliant Crystal Scarlet $6 R^{2}$ (an acid red 44) along with, as the last dye, a larger molecule than soluble blue (acid blue 22), namely Naphthalene Blue Black CS $^{3}$ (an acid black 41). It is to be noted that the effective or working size of a molecule may differ from its actual formula size (as shown structurally in volume 3 of the second edition of the Colour Index), depending presumably on the state of aggregation in the solvent used. The 44/41 Masson method revealed a curious concentration of the blue dye in middle-aged and old fibrin, while new fibrin and fibrin at the centre of larger masses of middle-aged fibrin gave a red colour. A further oddity of this method concerns the hyaline granules of renal tubules, in that within a single section one can find tubules in which granules of varied size are

${ }^{2}$ Messrs. L. B. Holliday Ltd.

${ }^{3}$ I.C.I. Ltd. all red, or are all blue, or are blue in the small an red in the larger, and these differences seem to be consistent within a zone; a variation was also seen $i \overrightarrow{\mathbb{H}^{3}}$ Paneth cells in the ileum that suggested an indicatiof of different physiological states. This is the only onfe of our fibrin methods that gives reasonably good staining of Negri bodies in formalin-fixed cerebella tissue (dark blue). The focal concentration of Naphthalene Blue Black, particularly in the fibrin im vascular walls, was found to be more specific afte pre-treating the section with alcoholic phosph $0_{-}^{-}$ tungstic acid, so we applied an orange dye isg alcoholic phosphotungstic as in Slidders' pituitar method, followed this with Naphthalene Blue Black to stain the fibrin, proceeded to aqueous phosphar tungstic acid, and finished with a large red dye, orfe of the direct reds (functionally large dyes that staiom cotton directly, that is without the need of $\vec{a}$ mordant). This orange-blue-direct-red method we originally designated OBDR45 later termed in the laboratory the Obadiah method, although it has a界 elegance and an effectiveness lacking in the bu值 described by Tristram Shandy.

The other principle we used was the one exemp官 fied by the phloxin-tartrazine method (Lendrum $1939,1947)$, in which, after staining with a red of the fluorescein group, differentiation is carriedsout by a slightly larger yellow dye dissolved in cellosầ (ethylene glycol mono-ethyl ether). The first improve ment on the original phloxin-tartrazine method wo the empirically discovered value of replacing tartro zine by Lissamine Flavine. ${ }^{4}$ While we were using this stain, it was discovered by chance that a treatment $\vec{f}$ the section for some 24 hours or more by a fat

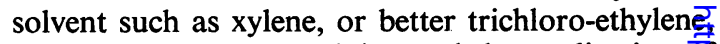
between the phloxin staining and the application of the yellow differentiator-staining solution, slowe down the differentiation accompanied by an i: creased contrast, i.e., stronger retention of phloxin 適. fibrin and readier removal of phloxin from the other materials in the section. We then found that the degreasing treatment (commercial trichloroethylene or Westrosol, which we use, is a well known dêgreaser in various trades) could be applied effees tively before the phloxin staining and with even further benefit by a brief additional period after the phloxin staining. This allowed us to use yellow dyes that without the degreasing treatment whipped of the phloxin speedily and indiscriminately. Thus, f $\mathrm{ft}^{\mathrm{t}}$ example, Dr. G. P. T. Barclay in his studies on early myccardial degeneration (1961) found that sequence of phloxin with pre- and post-degreasing and differentiation with a cellosolve solution of $\cdot \frac{}{a}$ milling yellow (large type of dye used in dyeing wogl that is going on to be wet-milled), gave very cleast 'FFS of I.C.I. Ltd. (acid yellow 7). 旅 
delineation of the early degenerative changes in myocardial fibres. These methods seem to work only with fluorescein reds, but Slidders found that acid magenta could be used as the red dye if it were followed by aqueous phosphotungstic acid; he then differentiated with a solution of a milling yellow in cellosolve and obtained in his fuchsin-miller method (1961a) a very useful means of staining fibrin, particularly in the study of the relation of fibrin to elastic tissue or reticulin.

Our latest variant of this red-yellow sequence uses one of the rhodamine dyes ${ }^{5}$ followed by differentiation with a cellosolve solution of a milling dye, Coomassie Fast Yellow GS $^{6}$ (acid yellow 85). This, especially in conjunction with the degreasing treatment, is a very selective method for the staining of some inclusion bodies at least, for example those in giant-cell pneumonia and in the lung of ferrets inoculated with canine distemper; it is also excellent for mast cell granules, Paneth cell granules, and the globular bodies of plasma cells. As a fibrin stain it demands some care in the differentiation, although we consider that the intracytoplasmic granules, which we think are engulfed fibrin, seen in pulmonary phagocytes and in the endothelium of arteries and veins containing thrombus, are better shown by this method than by any of the others.

Finally, it must be stated that the diagnostic utility of the methods described hereafter depends on obedience to the details of technique. We have no doubt that ambiguous appearances are at their rarest in material that has had the most careful fixation and been processed by the slow dehydration and double-embedding mentioned in the next section. The staining methods are of course at their best on fine sections, but the results even on excellent material can be completely upset if the times are disregarded, because the capability of localizing dyes of different molecular sizes in tissues of different polymolecular texture is a function of time. For example, a larger dye will, given time, work its way into a tight texture and eject meanwhile a smaller dye which in virtue of its smaller size had taken immediate possession of the site; thus whatever method is used for demonstration of fibrin or of granules carelessness will bring confusion. The times we commend were determined by 'a strict and pragmatic people, like the mass of the Scotch', and in relation to tissues prepared as advised.

\section{FIXATION AND THE STAINING OF FIBRIN}

The fixation that best demonstrates fibrin is a formalinmercuric chloride combination with prolonged fixation. If the tissue is fresh we immerse it directly in formol-

${ }^{5}$ Kiton Rhodamine B (acid red 52) manufactured by Messrs. Ciba. 'I.C.I. Ltd. corrosive $(900 \mathrm{ml}$. water, $30 \mathrm{~g}$. mercuric chloride, $100 \mathrm{ml}$. commercial formalin, plus a few millilitres of saturated aqueous acid magenta for identification). If, however, tissue is from a necropsy more than four hours old, fixation is begun in formol-saline and within five hours the tissue is transferred to $5 \%$ aqueous mercuric chloride; the preliminary fixation in formol-saline helps to preserve the erythrocytes (for discussion on this and 'formalin pigment' see Lendrum, 1949). Tissue originally fixed in formol-corrosive is transferred after 10 to 16 hours to $5 \%$ aqueous mercuric chloride.

The fixation of lung from necropsy demands special precautions if the situation of erythrocytes and exudates is to be maintained and the pulmonary structure is not to be crushed. Slices of lung of maximum thickness $1.25 \mathrm{~cm}$. (if human) are placed in formol-saline for two hours. The vacuum apparatus we use is the Gallenkamp vacuum oven horizontal model No. 7940; this has a reasonably large floor area to take flat dishes, and the slices of lung are taken of the largest possible area that the dishes permit. A thin sheet of absorbent cotton wool is laid on the slice to prevent the surface drying. Pressure is reduced to $20 \mathrm{~mm}$. and kept so for 10 minutes; it is then released very gently and the exhaustion is repeated two or three times within the two hours. The oven is set at $56^{\circ} \mathrm{C}$. as for paraffin embedding.

The formol-saline is now replaced by formol-corrosive and the material is left for 12 to 18 hours (overnight) on the bench. The slices are then cut to give the blocks that will be embedded; these can be half the thickness of the original slice and are trimmed so that the original outer surfaces will be the presenting surface at the microtome. These blocks are then placed in $3 \%$ aqueous mercuric chloride, resting as usual on cotton wool. If the blocks float, they are given vacuum treatment as before, and this is repeated until they stay sunk.

The secondary fixation in aqueous mercuric chloride is adequate for brain and liver in about seven to 10 days, kidney does well with two to three weeks, while heart and lung are the better of some six to eight weeks. A slow dehydration is followed, preferably, by double embedding (Lendrum, 1951); this is noticeably valuable on kidney by keeping the glomeruli in shape and flat on the slide, and on lung by keeping erythrocytes within the capillaries.

If tissue is already in paraffin and has been fixed in a chrome-containing fixative, it is possible to obtain some of the necessary de-chroming effect by treating the section for one hour before staining in a sulphurous acid solution prepared by bubbling sulphur dioxide from a syphon ${ }^{7}$ into $20 \%$ alcohol for five minutes (adapted from Mann, 1902). If formalin is deposited in the blood, this is removed, as is malarial pigment, by the use of absolute alcohol saturated with picric acid (Barrett, 1944; Lendrum, 1944); this needs some three hours, and there is probably some advantage in having $3 \%$ of mercuric chloride in this solution to improve the fixation.

In most cases a further general improvement in the final result is achieved by subjecting the section to degreasing; it is not possible to say beforehand when this should be done but it is certainly worth a trial in every case, and in some where the fixation had been poor ${ }^{\prime}$ British Drug Houses Ltd. 
the improvement is doubly welcome. Degreasing is most conveniently done immediately after dewaxing the section with xylene; it is then rinsed with Westrosol (commercial trichloroethylene) and left in a closed jar of Westrosol for 48 hours. It is then rinsed with absolute ethanol and either taken to water or treated with the picromercuric alcohol mentioned above; this latter should be done before the dewaxed section meets any water, and in the case of poorly fixed material should be for 24 hours. This is an adaptation of Leach's suggestion (1945) that alcohol fixation at this stage may rectify the failure of ordinary formalin fixation to penetrate fatty barriers. Our own impression is that fixation with mercuric chloride solutions as described above owes much of its value to the solubility of mercuric chloride in fat, and Leach's manoeuvre is neither necessary for nor effective on tissue fixed in this way, as it certainly is on tissue treated merely with formol-saline. After the picromercuric alcohol the section is hydrated, treated with iodine and hypo, and washed to remove the naked-eye evidence of picric staining.

\section{THE PICRO-MALLORY V METHOD FOR FIBRIN IN SECTIONS}

TECHNIQUE 1 Stain nuclei by the oxazine and haemalum sequence and rinse in tap water.

2 Yellow mordant for three to five min. and wash in tap water for one minute.

3 Stain with fuchsin for five min. and rinse in tap water.

4 Differentiate for 10 to $15 \mathrm{sec}$. and rinse in tap water.

5 Stain with phosphotungstic acid for five min. and rinse in tap water.

6 Stain with soluble blue (or light green) for two min. and rinse in tap water.

7 Dehydrate rapidly, clear, and mount in butyl phthalate styrene.

NOTES 1 The original celestin blue-haemalum sequence has been in continuous use for 21 years, and is the simplest way of obtaining a nuclear stain that resists picric and phosphotungstic acid. One may use either the commercially available form of celestin blue (mordant blue 14$)^{8}$ or another oxazine dye $^{9}$ (mordant violet 54 ). Both have proved excellent in routine use.

Allow $2.5 \mathrm{~g}$. iron alum to dissolve overnight at room temperature in $50 \mathrm{ml}$. distilled water: to this add $0.25 \mathrm{~g}$. of dye and boil for three minutes: filter when cool into a staining jar and add $7 \mathrm{ml}$. glycerol. This keeps for months. Stain for five minutes and rinse in tap water; filter on Mayer's haemalum for five minutes and wash.

2 The yellow mordant is $80 \%$ ethanol, saturated with picric acid $200 \mathrm{ml}$., orange $\mathrm{G} 0.4 \mathrm{~g}$., and Lissamine Fast Yellow $2 \mathrm{G},{ }^{10} 0.4 \mathrm{~g}$. The mordant deviates the fuchsin into fibrin and ideally keeps it out of the erythrocytes. If

${ }^{8}$ Supplied by M. Durand et Huguenin, S.A., Fabrikstrasse 40, Basle, Switzerland, as Coreine RR65.

'Available from I.C.I. Ltd., as Solochrome Prune AS.

${ }^{10} \mathrm{Lissamine}$ dyes are wool dyes produced by I.C.I. Ltd.: this one is acid yellow 17. used for more than five minutes it may produce some reddening of the nuclear staining.

3 Acid fuchsin or acid magenta (acid violet 19) is used as a $1 \%$ solution in $1 \%$ aqueous acetic acid.

4 The differentiator is $30 \mathrm{ml}$. of the yellow mordanto ( 2 above) with $70 \mathrm{ml}$. of $80 \%$ ethanol. Like No. 2 thiso keeps well. This solution helps to remove red from erythrocytes and keep it in the fibrin but its application should not be prolonged.

5 A $1 \%$ solution of phosphotungstic acid in water this 'fixes' the red in fibrin, and if the original fixation hap been satisfactory the other tissues relinquish the fuchsin:Dense tissues, such as hyaline collagen, are reluctant to $\vec{\omega}$ release the red, and this is particularly troublesome in poorly fixed material. In such a case the phospho tungstic action is prolonged to 10 minutes and the section is then treated with McFarlane's red differentiator (1944).

McFarlane's Stock Solution

Phosphotungstic acid .........25 g.

Picric acid ............. $2 \cdot 5 \mathrm{~g}$.

$95 \%$ ethanol $\ldots \ldots \ldots \ldots \ldots \ldots 100 \mathrm{ml}$.

McFarlane's Red Differentiator

Stock solution.............40 ml.

$95 \%$ ethanol ............40 ml.

Distilled water ............20 ml.

6 Soluble blue (acid blue 22) at $1 \%$ or light green SF (acid green 5) at $2 \%$ is used in $1 \%$ aqueous acetie acid. The larger dye direct blue $1^{11}$ may be substituted especially if a delicate stromal stain is wanted for high:power photography or to help in removing the tracesôt fuchsin from collagen; this dye works slowly.

7 Butyl phthalate styrene, the polystyrene mountan recommended by Kirkpatrick and Lendrum (1941) ir place of their original synthetic mountant D.P.X $\mathbb{D}$ (distrene, plasticizer, xylene), has preserved the colour of picro-Mallory sections with no deterioration over 18 years?

Mix $40 \mathrm{ml}$. dibutyl phthalate and $400 \mathrm{ml}$. xylene; dis? solve in this $120 \mathrm{~g}$. Styron. ${ }^{12}$

COMMENT The function of this method is not to. replace Masson's trichrome as such but to demon:strate fibrin and its relation to other tissues. Al though fibrin is the main retainer of the fuchsin, $\dot{B}$ strong affinity is shown by keratin, certain cyto plastic granules, some serous exudates with a high fat content, and a number of necrotic substances? Morphological distinctions should not be ignorew and this obligation does not remove the value of the method, as Attwood (1958) showed in his studies or amniotic embolism.

\section{MARTIUS-SCARLET-BLUE (MSB) METHOD: AN AUTOMATIC TRICHROME METHOD FOR STAINING FIBRIN}

This method has been evolved from the orange-fuchsin green method for the staining of the anterior pituitaryo

${ }^{11}$ Direct sky blue GS of Messrs. Ciba.

${ }^{12}$ Crystal clear K27, grade 666 gr 71 is obtained from Messrs. British Resin Products Ltd., Devonshire House, Piccadilly, London, W.1. (D) 


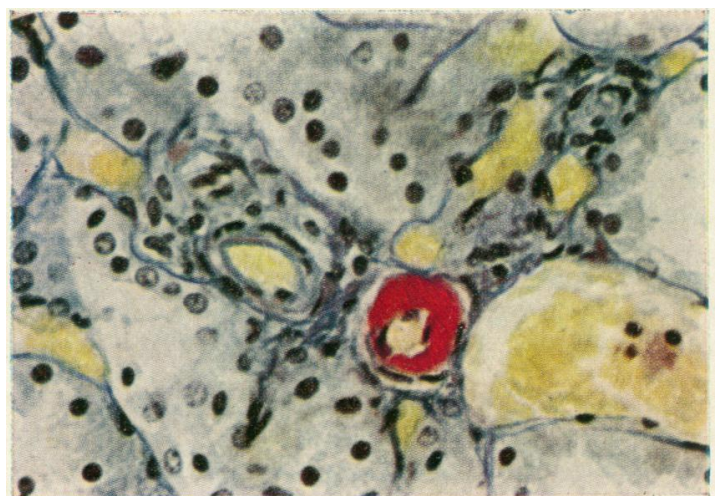

FIG. 1

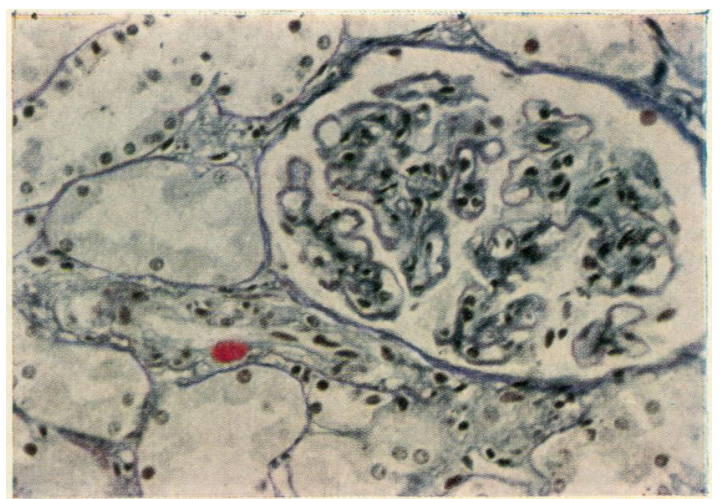

FIG. 2

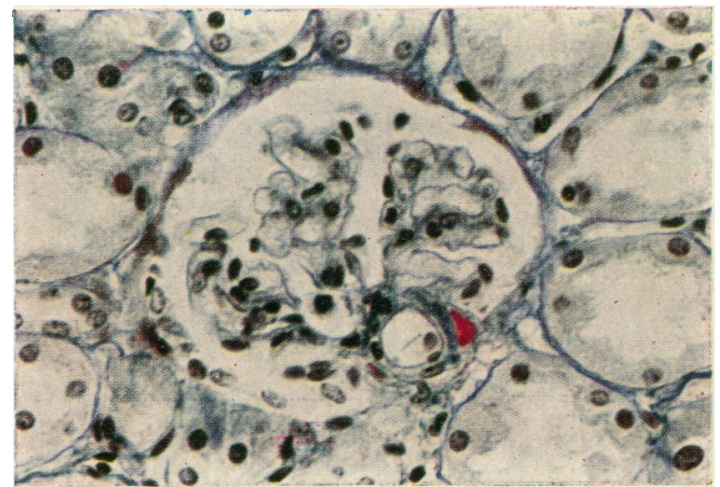

FIG. 3

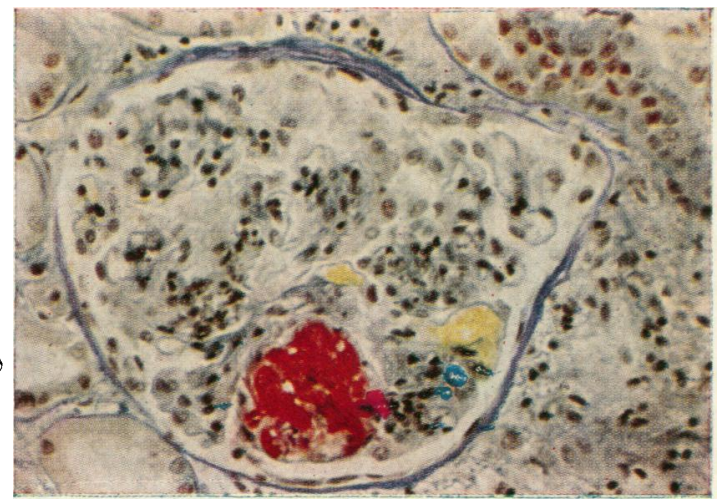

FIG. 5

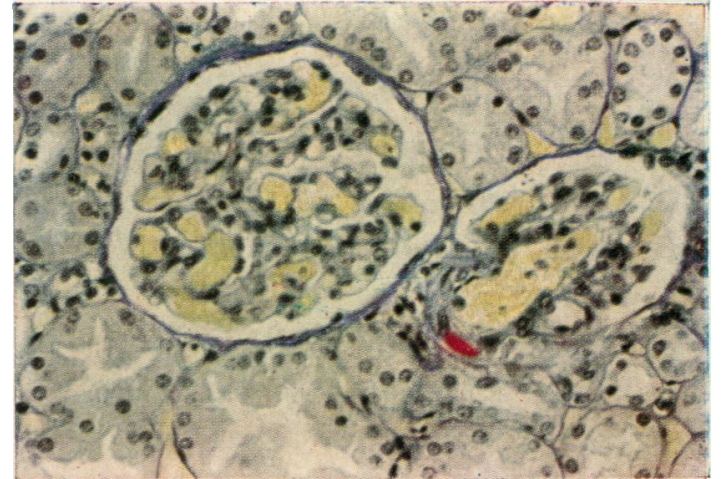

FIG. 4

FIG. 1. Stain: picro-Mallory. Renal arteriole with fibrin in wall, separated by endothelium from the lumen in which no fibrin is seen. Benign hypertension (N2016). $\times 333$.

FIG. 2. Stain: picro-Mallory. Glomerular arteriole showing a lozenge of red fibrin within the wall. Benign hypertension (A4325). $\times 213$.

FIG. 3. Stain: picro-Mallory. Glomerular arteriole showing lozenges of fibrin and acellular blue thickening (pseudo-collagen). Benign hypertension. $\times 333$.

FIG. 4. Stain: picro-Mallory. Glomerular arteriole with fibrinous lozenge. Girl, aged 11, death 16 hours after resection of aortic coarctation (N2605). $\times 213$.

FIG. 5. Stain: picro-Mallory. Focal glomerular lesion of the hyperergic form of plasmatic vasculosis. Streptomycin sensitization (N1596). $\times 213$.

All photographs were taken on $35 \mathrm{~mm}$. reversal film; the camera is a Leica combined with a Leitz Ortholux microscope. 


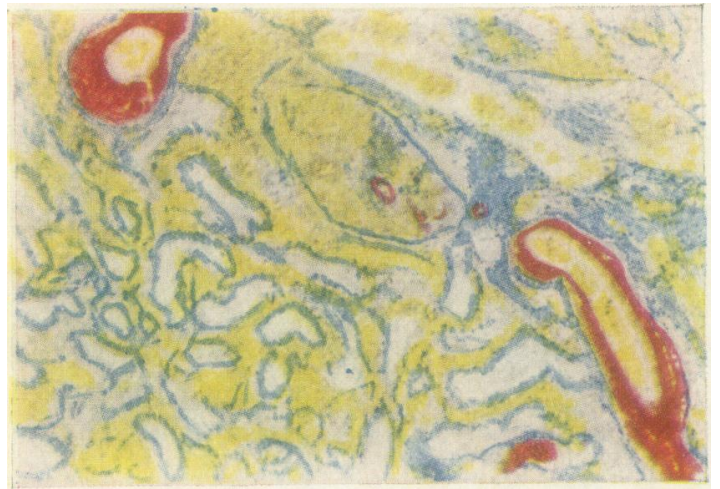

FIG. 6. Stain: MSB. Deposition of fibrin in the walls of arteriole, and glomerular capillaries, with notable absence of fibrin in the lumina and in the extravasated blood (erythrocytes yellow). Aseptic infarction of kidney (N4247). $\times 66$.

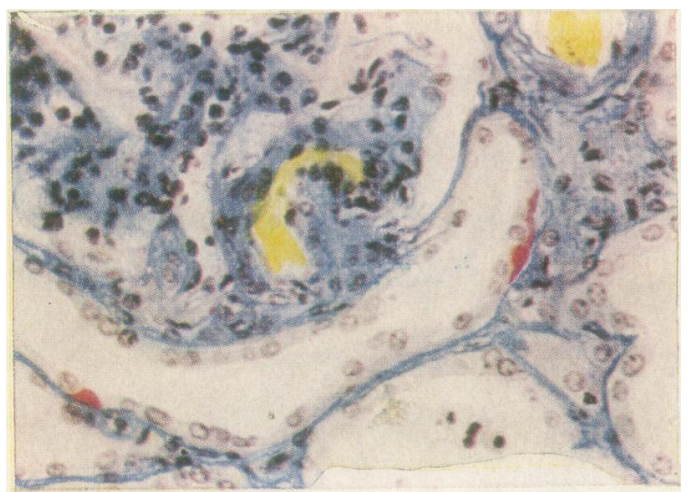

FIG. 7. Stain: MSB. Lozenges of fibrin in the tabule, between the epithelium and the basement membrane (blue, as is collagen). Diabetes mellitus (onset in maturity) with hypertension (N2400). $\times 266$.

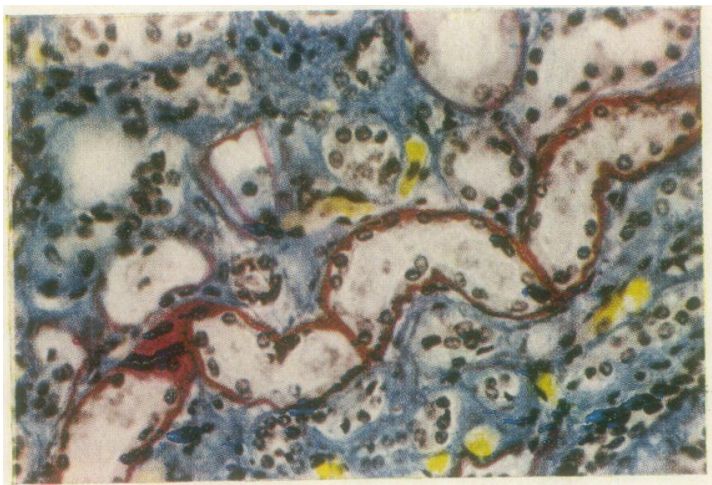

FIG. 8. Stain: MSB. The continuity of the layer of fibrin at the base of the epithelium suggests that the extravasated plasmatic exudate has wormed its way far from the blood vessel; amyloid deposit in some affected kidneys exactly mimics this disposition. Diabetes mellitus (N3400). $\times 213$.
(Slidders, 1961b). To obtain a good colour contrast between the erythrocytes and the fibrin, the first dye응 Martius yellow (acid yellow 24), was chosen on the grounds that it is a pure yellow and a small molecule. The red dye, Brilliant Crystal Scarlet $6 \mathrm{R}^{13}$, contrasts welto with the yellow, and although it has a slightly pooro affinity for the freshest fibrin it stains rather older fibrin than does the fuchsin of the picro-Mallory method. The method is more automatic than the other fibrin methods and with well-fixed tissue scarcely needs the degreasing manoeuvre (p. 404).

TECHNIQUE 1 Stain nuclei by oxazine and haemalum $\overrightarrow{\vec{\omega}}$ sequence (p. 406) and rinse in tap water.

2 Differentiate in $0.25 \% \mathrm{HCl}$ in $70 \%$ ethanol.

3 Wash well.

4 Rinse in $95 \%$ ethanol and stain with $0.5 \%$ Martius $\vec{G}$ yellow in $95 \%$ ethanol containing $2 \%$ phosphotungstic or acid for two minutes.

5 Rinse in water and stain with $1 \%$ Brilliant Crysta Scarlet $6 \mathrm{R}$ in $2.5 \%$ aqueous acetic acid for 10 minuces

6 Rinse in water and treat with $1 \%$ aqueous phos $-\overrightarrow{-}$ photungstic acid for five minutes.

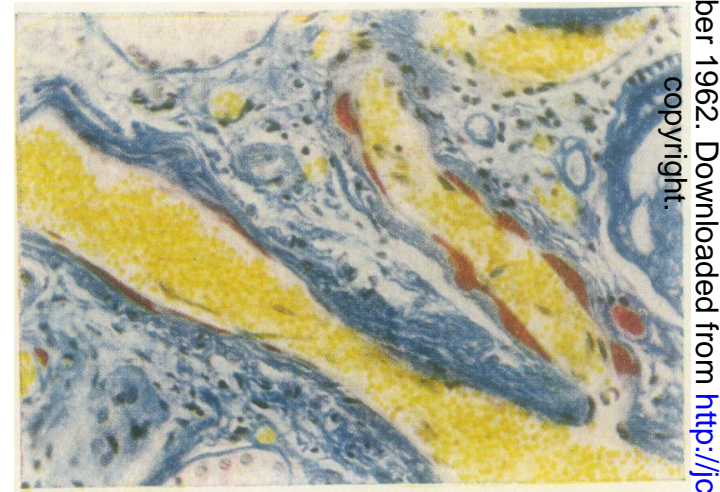

FIG. 9. Stain: MSB. The artery and its branch, character istically more involved near its origin, show the usual shape of the fibrin lozenges occurring in diabetes mellitus of the type whose onset is in maturity, flat toward the lumen an\& protecting into the wall. Diabetes mellitus $($ N3742). $\times 166$.

7 Rinse in water and stain with $0.5 \%$ soluble blue (acid blue 93) in $1 \%$ aqueous acetic acid for 10 minutes.

8 Rinse in water, mop the slide, and dehydrato directly by running on absolute ethanol, and so to xylene and mount in butyl phthalate styrene.

${ }^{13}$ Acid red 44 from Messrs. L. B. Holliday Ltd., Dyeworks, Hudder field.

\section{MSB}




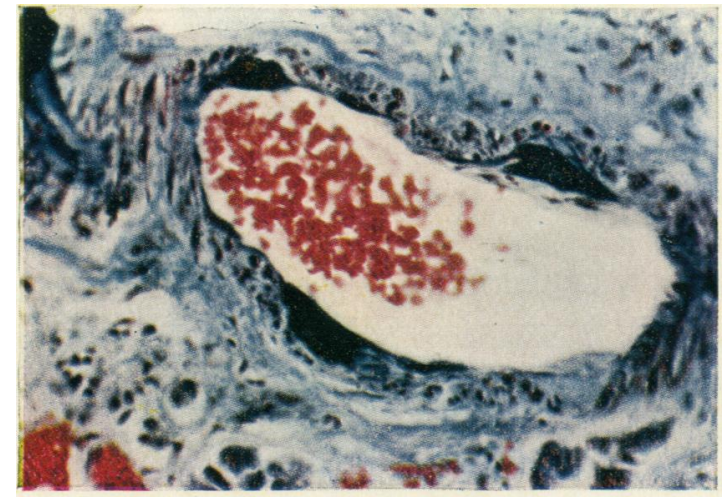

FIG. 10. Stain: 44/41 Masson. The intramural lozenges of fibrin, blue-black in colour, again show the outward projection. Diabetes mellitus with hypertension, onset in maturity (M335/57). × 213 .

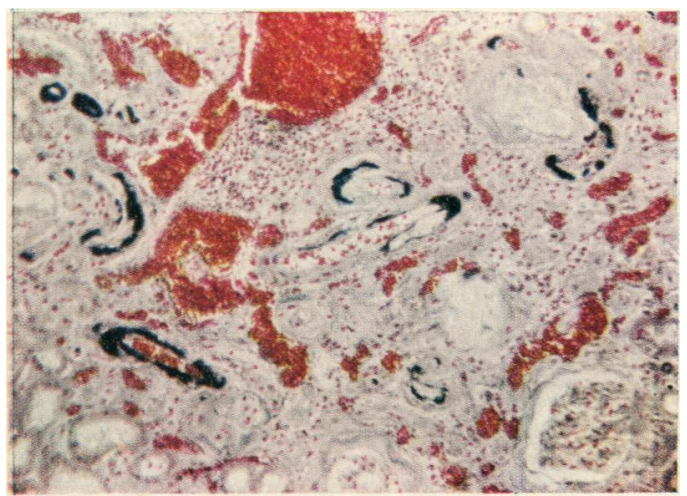

FIG. 11. Stain: 18/41 Masson. This lower power shows that the susceptibility of the renal arteries to the insudation of plasmatic substances can be widespread. Fibrin is blueblack. Diabetes mellitus, onset in maturity (N3743). $\times 66$.

\section{Masson 44/41}

MASSON $44 / 41$

TECHNIQUE 1 Remove wax with xylene, rinse with trichloroethylene, and immerse in a closed jar of trichloroethylene for 48 hours.

2 Rinse in absolute ethanol and immerse for three hours in a closed jar of absolute ethanol saturated with picric acid and containing $3 \%$ of mercuric chloride.

3 Bring to water, treating with iodine and hypo, and wash till picric staining is no longer visible to the naked eye.

4 Stain the nuclei by the oxazine and haemalum sequence (p. 406) and rinse in tap water.

5 Differentiate with $0.25 \% \mathrm{HCl}$ in $70 \%$ ethanol.

6 Wash well.

7 Stain with $1 \%$ Brilliant Crystal Scarlet $6 \mathrm{R}$ in $1 \%$ aqueous acetic acid for five minutes.

8 Rinse in water and treat with $1 \%$ aqueous phosphotungstic acid for five minutes.

9 Rinse in water and stain with $1 \%$ Naphthalene Blue Black CS in $1 \%$ aqueous acetic acid for 30 minutes.

10 Rinse in water, dehydrate in $95 \%$ and absolute ethanol, clear, and mount in butyl phthalate styrene.

NOTES 1 The degreasing and picric treatment are both essential to give the full clarity and contrast of the method.

7 The Brilliant Crystal Scarlet 6R (Messrs. Holliday's name for the acid red 44 they manufacture) was our original red, hence the name given to the method. Its replacement by an acid red 18, for example, Messrs. I.C.I.'s product Naphthalene Scarlet 4R, gives a more delicate result which is sometimes useful. Both are clear and pleasant substitutes for acid fuchsin in the classical Masson method and make it easier to obtain the transparency which is too often lost by skilled workers even on thin sections.

9 Naphthalene Blue Black CS is an acid black 41 manufactured by Messrs. I.C.I. Ltd. It has a fairly large molecule and stains collagen a pale blue; this happens slowly but there is a fairly rapid and intense blue-black staining of old fibrin. This is particularly obvious in the intramural deposits in the renal vessels of diabetes mellitus with hypertension. Fresh fibrin tends to hold the red dye and in between there is a purple-black stage: some confusion may arise from the retention of red in the centre of masses of dense fibrin.

COMMENT In the ileum with the original Masson method, both the Paneth and the enterochromaffin cell granules are red; with the 44/41 Masson the enterochromaffin granules are bright red but the Paneth granules are dark blue and only as they gain size do they show red retention. One case showed all the Paneth granules red, but it is not known if this is related to a stage in digestion or merely to some unrecognized technical flaw. The inclusions in plasma cells are bright red whereas in formalin-fixed cerebellum Negri bodies are blue-black.

\section{THE OBADIAH METHOD}

TECHNIQUE 1 Dewax the section and treat with trichloroethylene for 48 hours.

2 Treat with picromercuric alcohol for two hours, and bring to water.

3 Stain nuclei with oxazine-haemalum, differentiate, and wash.

4 Rinse in $95 \%$ ethanol and stain with $0.5 \%$ orange $G$, $2 \%$ phosphotungstic acid in $95 \%$ ethanol for two minutes.

5 Rinse in water and stain with $1 \%$ Naphthalene Blue Black CS in $1 \%$ aqueous acetic acid for up to 30 minutes.

6 Rinse in water and treat with $1 \%$ aqueous phosphotungstic acid for five minutes. 


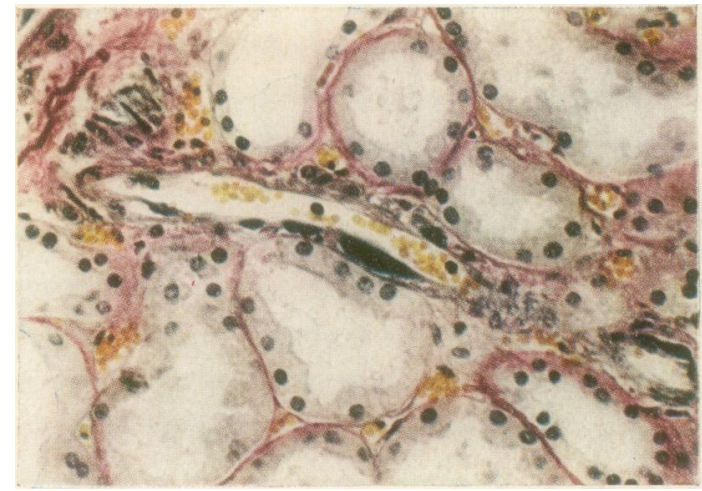

FIG. 12

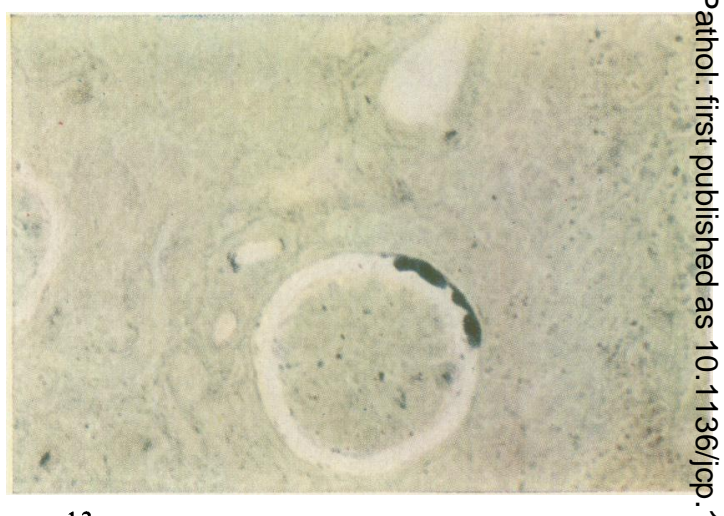

FIG. 13

FIG. 12. Stain: Obadiah. Renal arteriole with blue-black lozenges of fibrin in the wall. Diabetes $(N 3743)$. $\times 213 \pm$

FIG. 13. Stain: APTA Black, variant of Obadiah. Glomerulus with deposition of fibrin on the inner surface of the capsule. This varies in shape from one to another glomerulus, compressed flat against the capsule, bulging into the space or bosselated as here. Diabetes $($ M335/57). $\times 83$.

\section{Obadiah}

7 Rinse in water and stain with $2.5 \%$ Chicago red in $2.5 \%$ aqueous acetic acid for 15 to 30 minutes.

8 Rinse in water, mop the slide, and dehydrate directly by running on absolute ethanol, and so to xylene and mount in butyl phthalate styrene.

NOTES 1 Poor fixation generally causes irregular staining of the erythrocytes in that some of them take the black dye instead of the orange in this method (or the red in the 44/41 Masson).

4 Orange $\mathrm{G}$ (acid orange 10 ) is fairly sharply localized in the erythrocytes, young fibrin, and the more acidophilic intracytoplasmic inclusions and granules.

5 Naphthalene Blue Black CS (an acid black 41 manufactured by Messrs. I.C.I.) is of its nature a mixture with the special value to the pathologist that it stains old fibrin blue-black, particularly in the vessel walls in the kidney of hypertensive subjects with diabetes mellitus or with extreme tension, and of those with severe systemic lupus.

7 Chicago Red (a direct red 45 manufactured by Messrs. Geigy) is one of the large dyes that stain cotton directly, that is without a mordant; they stain collagen selectively but are slow in action. The final result is not unlike a delicate Van Gieson stain with the addition of the fibrin staining, and is of special value on thin sections of kidney. If one wishes more emphasis on the collagen and a staining of the finer reticulin, as in the glomeruli, a stronger red is available in acid red 130 (Polar Brilliant Red BN of Messrs. Geigy) which is used at $1 \%$ in $1 \%$ aqueous acetic acid (in place of the Chicago red) for 15 to 20 minutes.

8 Rapid dehydration with absolute ethanol (avoiding the use of diluted alcohols) is essential here, as it is with the MSB method and in the routine performance of Van Gieson's method.
VARIANT: A.P.T.A. BLACK A useful and simple scanning method to reveal the presence of plasmatic vasculosis is to follow the nuclear staining by a treatment with $\mathscr{S}^{\circ} \mathbb{D}$ phosphotungstic acid in $95 \%$ ethanol (no orange and then after a rinse, stain with the Naphthalene B⿳亠丷厂巾 Black solution as above. Then without counterstain itrg dehydrate, clear, and mount. The fibrinous deposit show up with an obvious opaque blue.

\section{THE YELLOSOLVE METHODS}

The origin of this type of method was the finding (Lendrum, 1939, 1947) that Masson's erythrosin-saffror? method could be replaced by a technique in which. phloxin was substituted for erythrosin, and tartrazine for the extract of saffron, if the tartrazine was used irp? cellosolve (ethylene glycol monothyl ether). It has now. been found that other substitutions have provide $\&$ more specific methods for fibrin staining (Yellosolve IP and for the staining of various intracellular bodies (Yellosolve II).

YELLOSOLVE I FOR FIBRIN 1 Dewax section and treat: with trichlorethylene for 24 to 48 hours.

2 Bring to water and stain for elastica if desired. If Sheridan's or another Weigert type of stain is used, cleap the background with $80 \%$ alcohol saturated with picric acid: if orcein is used and clearing should be necessar use acid alcohol.

3 Stain nuclei with oxazine and haemalum (p. 406 or haemalum alone, and wash well.

4 Stain with phloxin $0.5 \%$ in water containing $0.5 \%$ calcium chloride for 30 minutes.

5 Rinse in cellosolve and with tetrachloroethylen and immerse in tetrachloroethylene till next morning. 


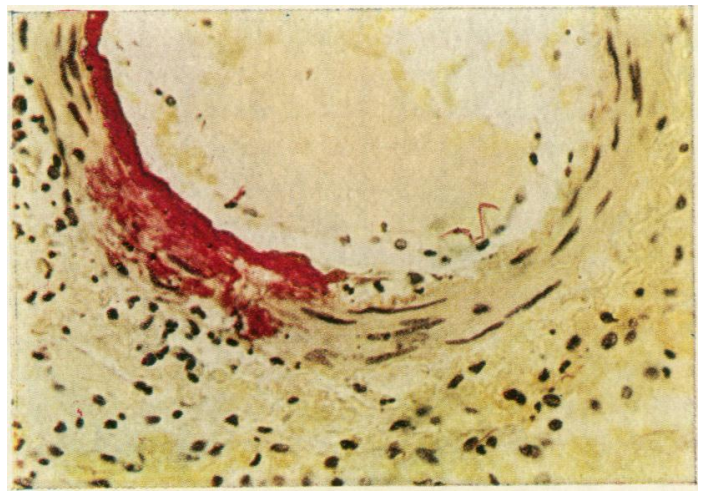

FIG. 14

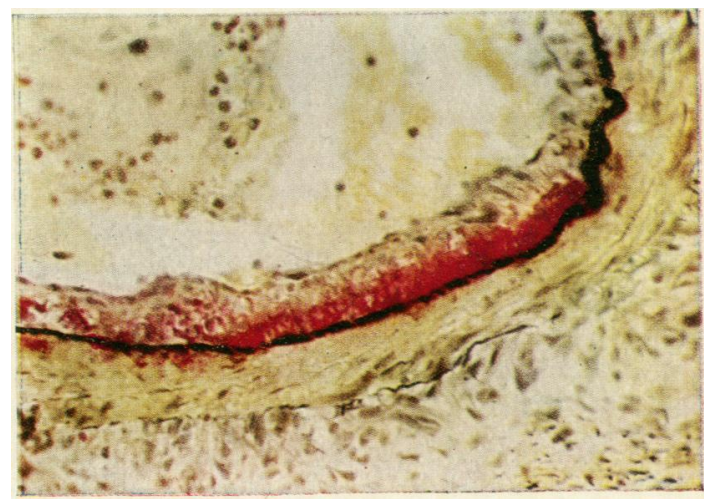

FIG. 16

FIG. 14. Stain: Yellosolve I with degreasing. Pulmonary artery showing plasmatic vasculosis, with the deposit of red fibrin revealing how the plasmatic proteins have penetrated into the wall. Infarction of lung (N2553). $\times 333$.

FIG. 15. Stain: Orcein for elastica Yellosolve I with degreasing. Pulmonary artery showing plasmatic vasculosis, with accumulation of fibrin in the intima; the internal elastica has been breached and the fibrin is 'straggling' across the media. Mitral stenosis with extreme pulmonary hypertension (A7729). $\times 213$.

FIG. 16. Stain: Weigert for elastica and Slidders' fuchsinmiller method. Artery from ileal region showing fibrin impacted on inner surface of internal elastica. Ileum resected two weeks after resection of aortic coarctation (Dr. Dallachy's case). $\times 213$.

FIG. 17. Stain:Weigert for elastica and Slidders' fuchsinmiller method. Pulmonary artery showing ruptured elastica, evidence of a previous episode of plasmatic vasculosis; the ring of fibrin round the current lumina is presumably indicative of a more recent phase of hypertension. Mitral stenosis with extreme hypertension (Dr. Florence McKeown's case). $\times 83$.

\section{Yellosolve I and II}

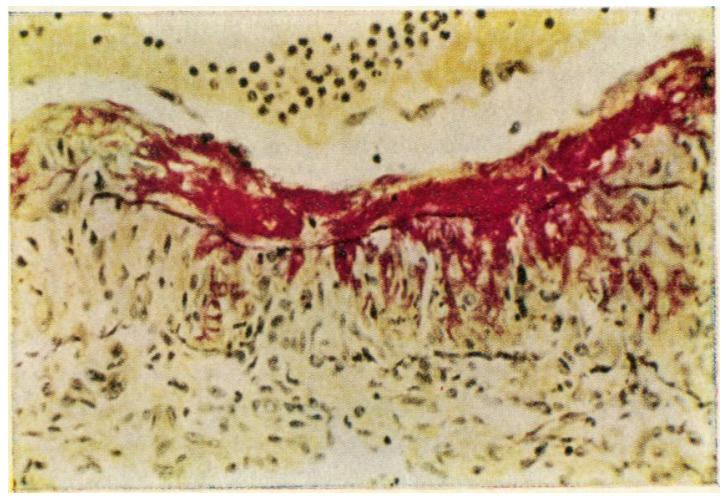

FIG. 15

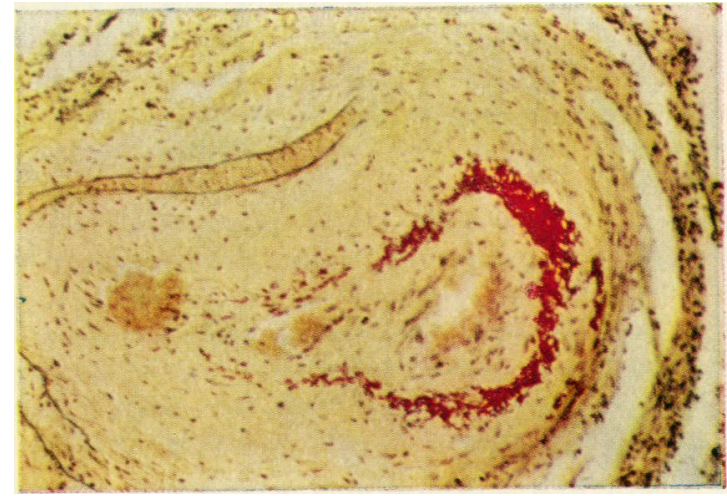

FIG. 17

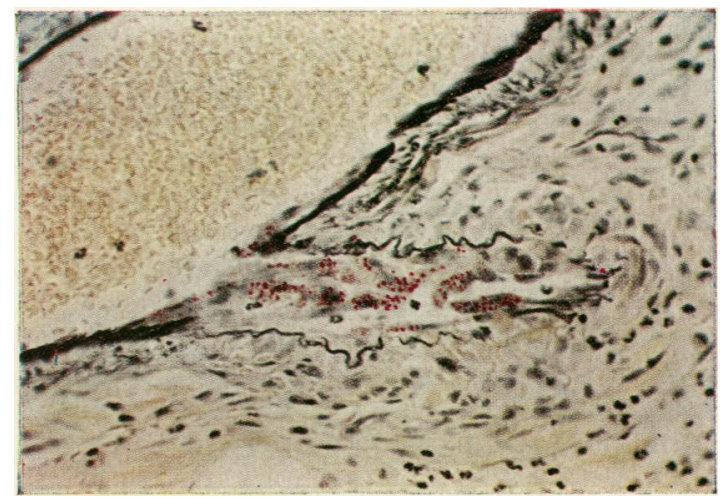

FIG. 18

FIG. 18. Stain: Weigert for elastica and Slidders' fuchsin-miller method. The endothelial cells of this ileal artery, particularly at the origin of the branch contain fine red-stained granules seen more commonly alongside thrombus. In this (same case as Fig. 16) there was intense congestion presumably secondary to hypertensive plasmatic vasculosis in the region. $\times 213$. 


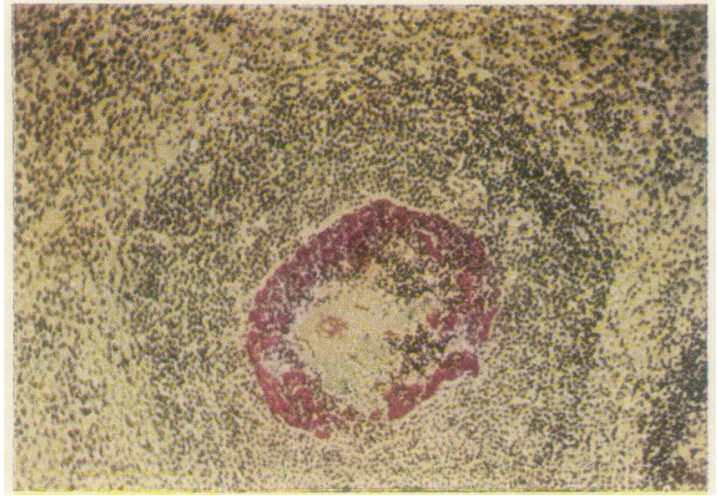

FIG. 19. Stain: Orcein for elastica and Yellosolve I with degreasing. Spleen showing fibrin deposition in a follicle; the distribution is the same as is shown by amyloid material in focal amyloidosis (sago spleen). This phenomenon was widespread in the spleen of this case of polyarteritis nodosa (from Dr. McInroy). $\times 83$.

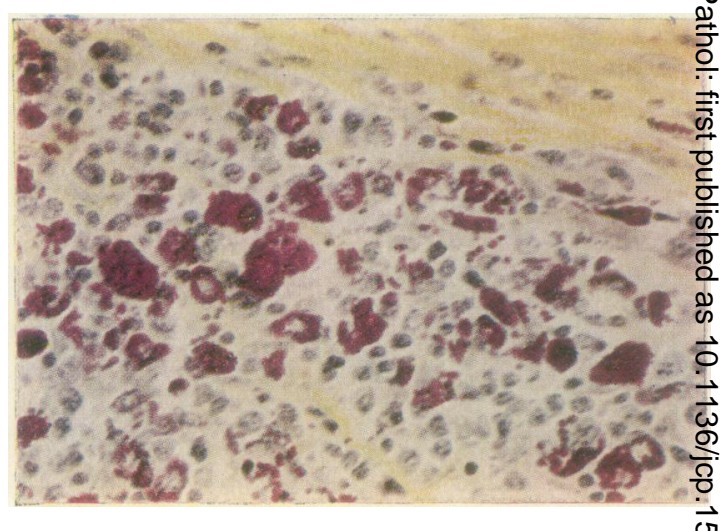

FIG. 20. Stain: Rhoda Coomassie with degreasing. OnE of many densely packed zones of plasma cells in thyroid gland with Hashimoto's disease (H.R. 2052/58). The intracytoplasmic inclusions have a strong affinity for the red dye. $\times 333$.

\section{Yellosolve I and II}

6 Rinse in cellosolve and differentiate in a saturated solution of Lissamine Flavine FFS in equal parts of cellosolve and ethyl phosphate, controlling microscopically.

7 Rinse in cellosolve, xylene thoroughly, and mount in butyl phthalate styrene.

NOTES 1 The degreasing procedure not only enhances the retention of phloxin in fibrin but has the curious and useful property of weakening the bond between phloxin and well-fixed erythrocytes.

2 This method shows some retention of phloxin in elastic tissues and any study of the relation of fibrin to elastica demands a separate staining of the elastica. Synthetic orcein is excellent (Chesterman and Leach, 1958 ); the stock solution of $1 \%$ in $80 \%$ alcohol is ripe in 24 hours and the working solution (life approximately 48 hours) can be prepared of 1 part stock with 9 parts of $80 \%$ ethanol containing $1 \% \mathrm{HCl}$. Possibly because of the absence of iron salts in this excellent stain for elastica, the subsequent counterstaining is delightfully transparent. The fuchsin-miller method of Slidders (1961a) is the only other fibrin method that we can recommend to follow staining of elastica.

3 The nuclear staining is not subjected to the action of phosphotungstic acid in this method, and therefore haemalum alone may be used, but there is quite a weakening effect produced by the acid dyes and therefore the time in haemalum should be increased, or the oxazine-haemalum sequence used with only slight differentiation in acid alcohol.

6 Lissamine Flavine FFS is Messrs. I.C.I.'s acid yellow 7. An American acid yellow 7 is made by the General Aniline and Film Corporation, Dyestuff and Chemical Division, New York, under the names
Fenazo Yellow XX, and Brilliant Sulpho Flavine FEA $\overrightarrow{0}$ The mordant yellow 33 (chemically similar to acid yelgov 7) supplied by MM. Durand et Huguenin, Fabrikstrasse 40, Basel, as Luxine Pure Yellow 6G, is slow in aonow but just as specific; if this dye is used without treatment with tetrachloroethylene and the time of dito ferentiation is kept short the result is comparable with the best examples of the old Masson's erythrosin-saffro technique.

COMMENT The method as described above with the full degreasing process shows a rapid removal of the phloxin from the collagen, from the erythrocytes. and largely from smooth muscle before there is an serious extraction of the phloxin from fibrin, keratin and various intracellular substances (mast ceE. granules, globular bodies of plasma cells, Paneth granules, coagulated myocardial sarcoplasm, hyali droplets of renal tubules, phloxinophil inclusions bo Variants of this method have been used by Attwoo (1958) to show keratin in amniotic embolism and bo Kirkland, Cass, Lowe, and Soutar (1959) to stain the granules in carcinoid tumour cells.

YELLOSOLVE II FOR INTRACELlular BODIES (Rhoc Coomassie Method) 1 Dewax the section and trese it with trichloroethylene for $\mathbf{4 8}$ hours.

2 Bring to water and stain with oxazine and hace malum.

3 Rinse in tap water and differentiate with $0.25 \% \mathrm{H}$ in $70 \%$ ethanol if necessary; wash well.

4 Stain with $1 \%$ Kiton Rhodamine B in $2.5 \%$ aqueous acetic acid for five minutes.

5 Rinse in cellusolve and differentiate in a saturate 
solution of Coomassie Fast Yellow GS in cellosolve; in properly fixed tissue the erythrocytes are cleared of all red dye before inclusion bodies show any loss of red.

6 Rinse in cellosolve, xylene, and mount in butyl phthalate styrene.

NOTES 4 Kiton Rhodamine B is an acid red 52 manufactured by Messrs. Ciba, known previously as Brilliant Kiton Red B, old C.I. 748; this is also used in the Kiton Red-Almond Green method for erythrocytes (Lendrum, 1949).

5 Coomassie Fast Yellow GS, and acid yellow 85 manufactured by Messrs. I.C.I., is a big dye of the milling type.

COMMENT This method is excellent for various intracytoplasmic inclusions although we have not been successful with Negri bodies. It is not recommended as a fibrin stain for general use although it is particularly good for the intracytoplasmic bodies in phagocytes that we believe are engulfed fibrin (Lendrum, 1961a); other granules that are distinctively shown include those of mast cells, plasma cells, and Paneth cells. Charcot-Leyden crystals in sections may retain the phloxin strongly even though the adjacent eosinophils fail to do so.

\section{CONCLUSION}

A study of staining methods giving in sections a sharp contrasty delineation of the material recognized in the past as fibrin has led to the view that fresh fibrin has a 'fine structure' capable of holding acid dyes with small molecules, and that as the fibrin ages its fine structure becomes more open and thus more capable of holding acid dyes with larger molecules.

The application of these new and more definitive methods to the study of the substance in the walls of blood vessels and in the tissues around such vessels, described in the past as fibrinoid, has shown an exactly similar response to dye as that of fibrin, and thus by the mere weight of coincidence we feel justified in calling it fibrin. Furthermore, it is possible in the absence of infective processes, with their fibrinolytic activities, to observe a longer duration of life in this fibrin and to see it change gradually to an acellular substance that now has a capacity for retaining dye equivalent to that of collagen. Two of the new methods are capable of distinguishing from this pseudo-collagen fibrin of an age that no longer reacts as fibrin. with the classical methods. Thus the definition of fibrin as studied in sections of tissue should perhaps include mention of the methods used in the process, and indeed it is probable that the methods of fixation and embedding we describe above are an essential component in obtaining clearcut results.

On a purely practical level we recommend for general use in the demonstration of fibrin the MSB method; it has an almost automatic character and by staining both fibrin and collagen with visually strong but different colours it shows the relationship with clarity. When the relation of fibrin to elastic tissue is to be shown, we would use the Yellosolve I method, or Slidders' fuchsin-miller method. To show older fibrin the best method is the 44/41 Masson. For high-power colour photomicrography on very thin sections of well-fixed tissue the gifted technician can obtain with picro-Mallory $\mathrm{V}$ a demonstration that will please both eye and mind.

We acknowledge gratefully the many gifts by colleagues in pathology in Dundee and elsewhere of material for study, also the generous help with information and sample dyes by the various manufacturers mentioned in the text; our special thanks go to Mrs. Neal, Academic Relations Department of Messrs. I.C.I. Ltd., for consistent aid.

The publication of the coloured plates was made possible by generous financial aid from the Carnegie Trust for the Universities of Scotland, Mrs. H. S. Johnston's Trust, and the Publishers. We are very conscious of our debt to them and to the blockmakers.

\section{REFERENCES}

Attwood, H. D. (1958). J. Path. Bact., 76, 211.

Baker, J. R. (1958). Principles of Biological Microtechnique, p. 240. Methuen, London.

Barclay, G. P. T. (1961). M.D. thesis, Edinburgh.

Barrett, A. M. (1944). J. Path. Bact., 56, 135.

Chesterman, W., and Leach, E. H. (1958). Quart. J. micr. Sci., 99, 65.

Crawford, T., and Woolf, N. (1960). J. Path. Bact., 79, 221.

Kirkland, J. A., Cass, R., Lowe, K. G., and Soutar, S. F. (1959). J. clin. Path., 12, 362.

Kirkpatrick, J., and Lendrum, A. C. (1941). J. Path. Bact., 53, 441.

Leach, E. H. (1945). Ibid., 57, 149.

Lendrum, A. C. (1939). Ibid., 49, 590.

- (1944). Brit. med. J., 2, 44.

(1947). J. Path. Bact., 59, 399

(1949). Ibid., 61, 443.

- (1951). In Recent Advances in Clinical Pathology, edited by S. C. Dyke, 2nd ed., pp. 513-544. Churchill, London.

(1955). J. clin. Path., 8, 180.

(1956). In Pulmonary Circulation and Respiratory Function, A symposium at Queen's College, Dundee, University of St. Andrews, p. 23. Livingstone, Edinburgh.

(1961a). J. clin. Path., 14, 325.

(1961b). Ned. T. Geneesk., 105, 1359.

McFarlane, D. (1944). Stain Tech., 19, 29.

Mann, G. (1902). Physiological Histology, p. 72. Clarendon Press, Oxford.

Morgan, H. G. (1952). Edinb. med. J., 59, 261.

Slidders, W. (1961a). J. med. Lab. Technol., 18, 36.

- (1961b). J. Path. Bact., 82, 532.

Venkataraman, K. (1952). The Chemistry of Synthetic Dyes, vol. 2, p. 1266. Academic Press, New York. 